EPJ Web of Conferences 111,02001 (2016)

DOI: 10.1051/epjconf/201611102001

C) Owned by the authors, published by EDP Sciences, 2016

\title{
Recent Developments in Nuclear Data Measurement capabilities at the Gaerttner LINAC Center at RPI
}

\author{
Y. Danon ${ }^{1, a}$, A. Daskalakis ${ }^{1}$, B. McDermott ${ }^{1}$, N. Thompson ${ }^{1}$, A. Youmans ${ }^{1}$, R. Block ${ }^{2}$, D. Barry ${ }^{2}$, \\ B. Epping ${ }^{2}$, G. Leinweber ${ }^{2}$, M. Rapp ${ }^{2}$, and T. Donovan ${ }^{2}$ \\ ${ }^{1}$ Gaerttner LINAC Center Rensselaer Polytechnic Institute, Troy, NY, 12180, USA \\ ${ }^{2}$ Bechtel Marine Propulsion Corporation, Knolls Atomic Power Laboratory, P.O. Box 1072, Schenectady, NY \\ 12301, USA
}

\begin{abstract}
The Gaerttner LINAC Center at RPI uses a $60 \mathrm{MeV}$ electron linear accelerator to produce short pulses of neutrons with duration of 5-5000 ns. The main research thrust at the Center is nuclear data for nuclear reactors and criticality safety applications. The Center includes several setups for time-of-flight measurements including neutron transmission, capture and scattering detectors, and a lead slowing-down spectrometer. Experiments were designed to produce neutron interaction cross sections that cover the energy range of $0.01 \mathrm{eV}$ to $20 \mathrm{MeV}$. Recently added experiments include: setups for $\mathrm{keV}$ and fast neutron transmission, a $\mathrm{C}_{6} \mathrm{D}_{6}$ detector array for $\mathrm{keV}$ neutron capture measurements, and a fast neutron scattering system. Results discussed here include fast neutron scattering and angular distributions for ${ }^{\text {nat }} \mathrm{Fe}$, iron capture measurements for incident neutrons from $1 \mathrm{keV}$ to $2 \mathrm{MeV}$, fast neutron transmission through $\mathrm{W}$ and $\mathrm{H}_{2} \mathrm{O}$ samples, and $\mathrm{keV}$ transmission through Mo isotopes.
\end{abstract}

\section{Introduction}

The Gaerttner LINAC Center at RPI utilizes a $60 \mathrm{MeV}$ electron Linear Accelerator (LINAC) to produce short pulses of neutrons used for time-of-flight measurements of nuclear data [1]. Recent developments include measurement setups for $\mathrm{keV}$ and fast neutron transmission, fast neutron scattering, and $\mathrm{keV}$ neutron capture. Each setup is a combination of a neutron production target with a detector array and the LINAC operated at appropriate conditions. These new capabilities provide new accurate experimental data that can help improve current evaluations.

\section{Experimental Setups}

Table 1 gives a list of the recently added experimental capabilities discussed here. Each setup uses its own data acquisition system, and some experiments on different flight paths can be performed simultaneously and thus better utilize the LINAC operation time.

\footnotetext{
ae-mail: danony@rpi.edu
} 
Table 1. Summary of recently added capabilities.

\begin{tabular}{llll}
\hline Setup & Target & Detector & Flight path \\
\hline keV Transmission & Pacman [2] & Li-Glass Array [3] & $100 \mathrm{~m}$ \\
Fast Transmission & Bare bounce [2] & EJ-301 array [4] & $250 \mathrm{~m}$ \\
Fast Scattering & Bare Bounce [2] & EJ-301 array [4] & $30 \mathrm{~m}$ \\
keV Capture & Pacman [2] & C $_{6}$ D $_{6}$ array [5] & $45 \mathrm{~m}$ \\
\hline
\end{tabular}

\section{Results}

Examples of recent experimental results utilizing the configurations shown in Table 1 are reviewed next:

\subsection{Fast neutron transmission}

The setup of this detector was described in detail in reference [6] where the detector was located at the $100 \mathrm{~m}$ flight station. For the measurements described here the detector was located at a $250 \mathrm{~m}$ flight station utilizing two detector modules. For this type of experiment, the LINAC typically operates with a pulse width of 6-8ns and repetition rate of 400 pulses/s. Recent experiments include transmission of ${ }^{\text {nat }} \mathrm{Pb}$, ${ }^{\text {nat }} \mathrm{W}$ and $\mathrm{H}_{2} \mathrm{O}$. The measured transmission of ${ }^{\text {nat }} \mathrm{W}$ is shown in Figure $1 \mathrm{left}$, and provides a good example of the capabilities of the system to help resolve differences between evaluations and provide new experimental data where none existed before. Above $6 \mathrm{MeV}$ the experimental data agree well with the experimental data of reference [7] and with the ENDF/B-VII.1 [8] evaluation. Below 6 $\mathrm{MeV}$ there is a discrepancy between the evaluations and also with the RPI experimental data.

One of the materials evaluated in the CIELO [9] project is ${ }^{16} \mathrm{O}$. Previously, Cerjacks measured the total cross section of oxygen in two experiments; the first was published in 1968 (CI68) [10]. The second paper 1980 (CI80) [11] mentions that the experiment was normalized to the 1968 experiments at 3.5 MeV. However, the EXFOR data do not seem to include this normalization. The CI80 experiment is the highest energy resolution experiment for ${ }^{16} \mathrm{O}$, and thus it is important to clarify its normalization. A high accuracy transmission experiment was designed at RPI to resolved this normalization issue using water samples and using the ENDF/B-VII.1 evaluation to correct for the $\mathrm{H}_{2}$ in the sample. The experiment was designed such that at the minima of the ${ }^{16} \mathrm{O}$ cross section near 2.35 $\mathrm{MeV}$, the cross section of $\mathrm{H}_{2}$ is measured. This serves as a verification of the normalization of the current transmission experiment. Figure 1 right shows the results of this measurement. It was found that for the energy range of $3.2 \mathrm{MeV}<\mathrm{E}<6 \mathrm{MeV}$ the ratio of the CI80 data to the RPI experiment was $0.968 \pm 0.002$ where the ratio of the CI68 data to the RPI experiment was $1.009 \pm 0.002$. At the ${ }^{16} \mathrm{O}$ minima near $2.35 \mathrm{MeV}$ the ratio of the $\mathrm{H}_{2} \mathrm{O}$ to $\mathrm{H}_{2}$ transmission was $0.996 \pm 0.003$. The energy resolution of the new RPI measurement is lower than the CI80 experiment but higher than the CI68 experiment. The finding for the CI80 experiment supports the normalization used in reference [12] (reduction of $3.5 \%$ ) to agree with the Johnson experiment [13].

\subsection{Mid energy (keV) transmission}

The Mid-energy transmission detector setup was described in detail elsewhere [14]. This detector uses an array of four Li-glass detector modules located at $100 \mathrm{~m}$ flight path. It was designed to cover the energy range from a couple of $\mathrm{keV}$ to $0.5 \mathrm{MeV}$, which includes resolved and unresolved regions. The results of measurements of Mo isotopes can be found in references [14] and [15]. An example of the 

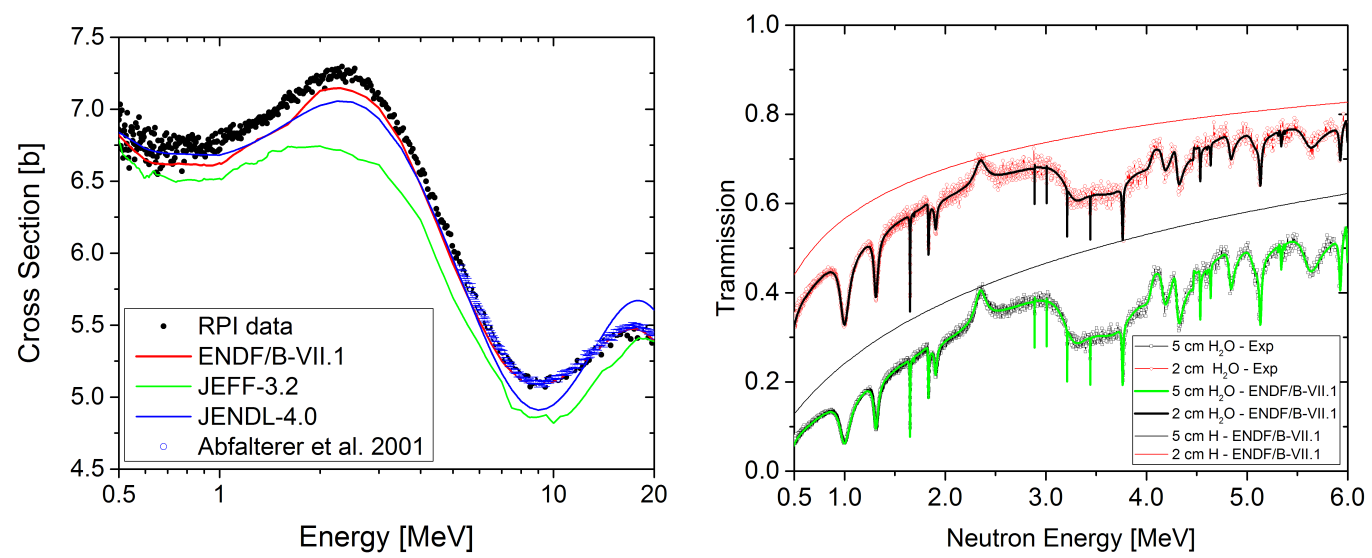

Figure 1. Left-Comparison of the total cross section of ${ }^{\text {nat }} \mathrm{W}$ with evaluations and with the data from reference [7]. Right- transmission through $\mathrm{H}_{2} \mathrm{O}$ samples and the ENDF/B-VII.1 evaluation (constructed for $\mathrm{H}_{2} \mathrm{O}$ ). The measured transmission near $2.35 \mathrm{MeV}$ matches the evaluated transmission of $\mathrm{H}_{2}$ which is used as validation of the normalization.

transmission of Mo isotopes is shown in Figure 2 with the ENDF/B-VII.0 evaluation. The new data show resonance structure that is not in the current evaluation and can be used to revise the evaluation. The data were used for unresolved resonance analysis in reference [15].

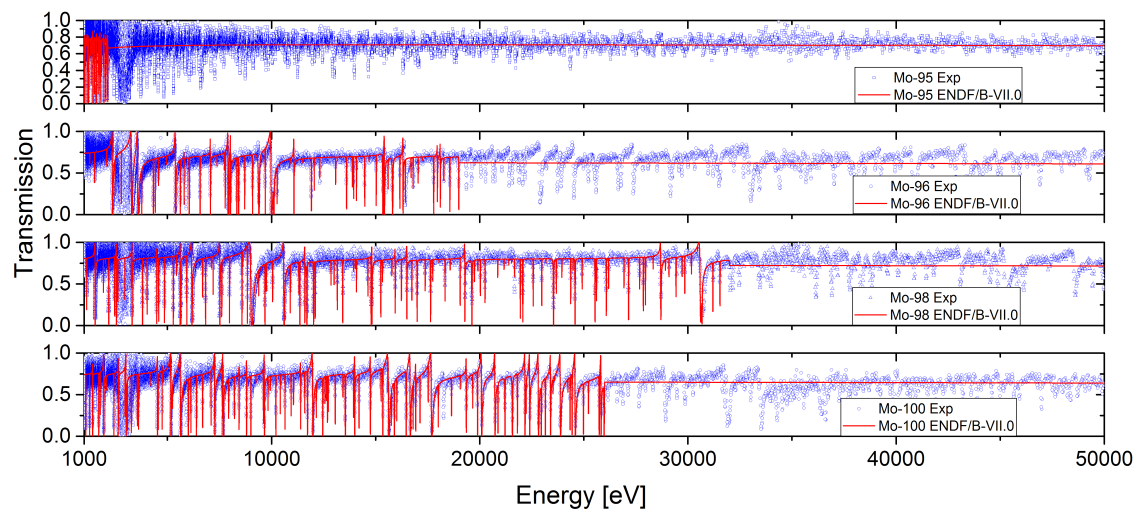

Figure 2. Measured transmission of ${ }^{95,96,98,100}$ Mo and the ENDF/B-VII.0 evaluation.

\subsection{Mid energy (keV) capture}

An array of four $\mathrm{C}_{6} \mathrm{D}_{6}$ detectors was constructed at a dedicated $45 \mathrm{~m}$ flight station. This setup was designed to extend the upper energy of capture measurements from $\approx 2 \mathrm{keV}$ to $\approx 2 \mathrm{MeV}$. The $\mathrm{C}_{6} \mathrm{D}_{6}$ detector concept is well established, see for example [16], and was chosen because it provides low 
sensitivity to scattered neutrons. However, in order to reduce the sensitivity to variations in the capture gamma cascade spectra and to account for the detection efficiency, a weighting function is needed. To provide all the data necessary for applying a weighting function and for flexibility of the data analysis, all 4 detectors were digitized with 10 bit pulse height resolution and $0.8 \mathrm{~ns}$ timing. To process the data a new multithreaded data acquisition system and data processing codes were written. The first measurements with this system were for ${ }^{\text {nat }} \mathrm{Fe}$ and Ta samples. A measurement of ${ }^{56} \mathrm{Fe}$ was also recently completed. Fe has a capture cascade that is dominated by high energy gamma rays $(\approx 7 \mathrm{MeV})$ which allows discrimination of the gammas from first inelastic state. Previous experimental data are available up to $200 \mathrm{keV}$, and one set by Spencer (measured at ORELA) that was not published is available up to $600 \mathrm{keV}$. Above this energy only a few experimental points exist. An experiment with ${ }^{\text {nat }} \mathrm{Fe}$ was performed, and a dedicated weighing function was used for the analysis. The neutron flux shape was measured using a $\mathrm{B}_{4} \mathrm{C}$ sample. The experimental results, evaluations, and other experimental data are shown in Figure 3. For comparison with other experiments both the RPI experimental capture yield and MCNP simulations were converted to cross sections using the thin sample approximation. Above $847 \mathrm{keV}$ inelastic gammas were measured but can be discriminated as illustrated in Figure 3. Multiple scattering was estimated using MCNP. Above $850 \mathrm{keV}$ the new data is in good agreement with the evaluations and other available experiments. Above the first inelastic state of ${ }^{54} \mathrm{Fe}$ at about $1.4 \mathrm{keV}$ the new data is substantially lower than the current evaluations. A capture measurement of ${ }^{56} \mathrm{Fe}$ was performed (analysis in progress) which might help resolve this issue.

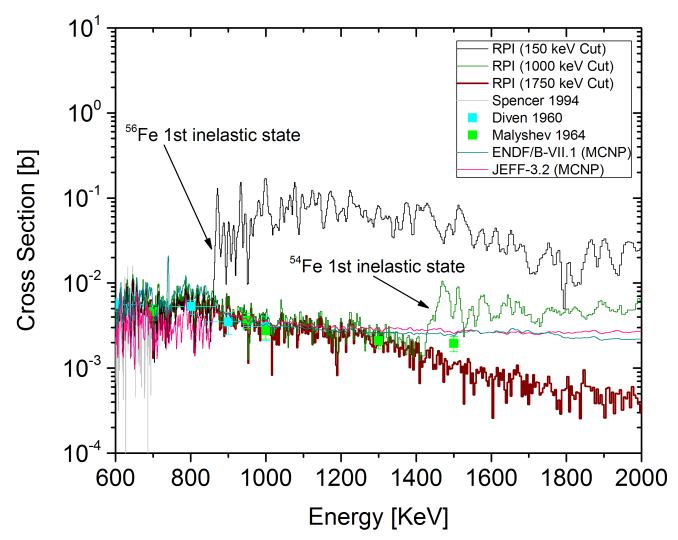

Figure 3. Neutron capture on $20 \mathrm{~mm}$ thick ${ }^{\text {nat }} \mathrm{Fe}$ sample. Above $600 \mathrm{keV}$ the plots also illustrate the increase in count rate due to inelastic gammas that was eliminated by increasing the pulse integral discriminator settings.

\subsection{Fast neutron scattering}

The neutron scattering detector array was previously described in detail [4]. Recently the data analysis codes and methodology were improved [17]. This includes better pulse shape classification for gamma rejection and implementation of a gamma rejection technique for high gamma rates. These developments enable neutron scattering measurements in the presence of high gamma background such as for ${ }^{238} \mathrm{U}$ and ${ }^{235} \mathrm{U}$ where fission is occurring and producing multiple gammas. Although this system is referred to as a neutron scattering system, it measures all the neutrons scattered or emitted from the sample. The experimental data were then compared to MCNP simulations that included 
detailed experimental parameters such as geometry, incident neutron flux, and detector efficiencies. This method provides a benchmark to compare how different nuclear data evaluations perform. For the case of $\mathrm{Fe}$ a method was developed to separate elastic from inelastic scattering. This is possible because of the relatively high energy levels for inelastic scattering which results in a large difference between elastically and inelastically scattered neutron. The scattered neutron energy can thus be inferred from the detected pulse shape. As an example the results for a scattering angle of 153 deg are shown in Figure 4. A comparison of the experiment and evaluations in Figure 4 indicates that for some time-of-flight regions the JEFF-3.2 [18] and JENDL-4.4 [19] evaluations are closer to the experiment compared to ENDF/B-VII.1. The ratio of inelastic to elastic scattering is in good agreement with the evaluations. For some of the points ENDF/B-VII.1 seems slightly higher than the other evaluations and the experimental data.
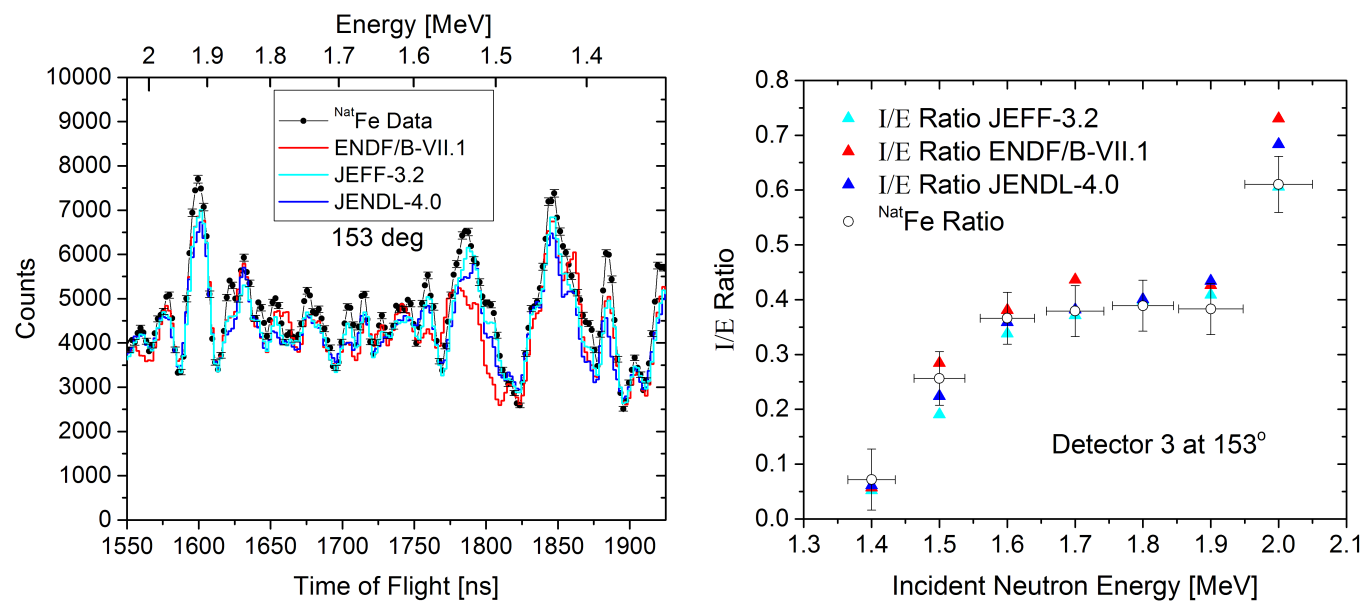

Figure 4. Neutron scattering from ${ }^{\text {nat }} \mathrm{Fe}$ sample. The left plot shows the data in TOF (energy scale is on the top $\mathrm{x}$-axis) compared with MCNP simulations using different evaluations. The right plot shows the ratio of inelastic to elastic scattering.

\section{Summary and Conclusions}

The Gaerttner LINAC Center at RPI added new capabilities, and some of them are presented here. Fast neutron transmission of $\mathrm{W}$ shows large deviations between the evaluations and the RPI experiment near $2.5 \mathrm{MeV}$. Fast neutron transmission through $\mathrm{H}_{2} \mathrm{O}$ provides new high resolution data for ${ }^{16} \mathrm{O}$ below $3.2 \mathrm{MeV}$ where the CI80 starts. The measurement also established that the CI80 [11] is about $3.2 \%$ lower than the CI68 [10]. The current experiment uses the unique minima in the ${ }^{16} \mathrm{O}$ cross section as an anchor for the normalization of the data. A mid energy Li-Glass detector array enabled new transmission measurements on several Mo isotopes. The data showed resolved and unresolved resonance structure that can help improve the evaluations. New capture measurements of Fe in the $\mathrm{keV}$ and $\mathrm{MeV}$ incident neutron energy regions were enabled by a new $\mathrm{C}_{6} \mathrm{D}_{6}$ detector array. 


\section{References}

[1] Y. Danon, R. Block, R. Bahran, M. Rapp, F. Saglime, C. Romano, J. Thompson, D. Barry, N. Drindak, J. Hoole et al., Proceedings of The Eighth International Topical Meeting on Nuclear Applications and Utilization of Accelerators(AccApp 07) (2007)

[2] M. Overberg, B. Moretti, R. Slovacek, R. Block, Nuclear Instruments and Methods in Physics Research Section A: Accelerators, Spectrometers, Detectors and Associated Equipment 438, 253 (1999)

[3] R. Bahran, Y. Danon, M. Rapp, D. Williams, D. Barry, G. Leinweber, R. Block, J. Hoole, Proceedings of the Tenth International Topical Meeting on Nuclear Applications of Accelerators (AccApp 2011) (2011)

[4] F. Saglime, Y. Danon, R. Block, M. Rapp, R. Bahran, G. Leinweber, D. Barry, N. Drindak, Nuclear Instruments and Methods in Physics Research Section A: Accelerators, Spectrometers, Detectors and Associated Equipment 620, 401 (2010)

[5] Y. Danon, L. Liu, E. Blain, A. Daskalakis, B. McDermott, K. Ramic, C. Wendorff, D. Barry, R. Block, B. Epping et al., Transactions of the American Nuclear Society, Vol. 109, p. 897-900, Washington, D.C (2013)

[6] M.J. Rapp, Y. Danon, F.J. Saglime, R.M. Bahran, D.G. Williams, G. Leinweber, D.P. Barry, R.C. Block, Nuclear Science and Engineering 172, 268 (2012)

[7] W.P. Abfalterer, F.B. Bateman, F.S. Dietrich, R.W. Finlay, R.C. Haight, G.L. Morgan, Phys. Rev. C 63, 044608 (2001)

[8] M. Chadwick, M. Herman, P. Obložinský, M. Dunn, Y. Danon, A. Kahler, D. Smith, B. Pritychenko, G. Arbanas, R. Arcilla et al., Nuclear Data Sheets 112, 2887 (2011), special Issue on ENDF/B-VII.1 Library

[9] M. Chadwick, E. Dupont, E. Bauge, A. Blokhin, O. Bouland, D. Brown, R. Capote, A. Carlson, Y. Danon, C.D.S. Jean et al., Nuclear Data Sheets 118, 1 (2014)

[10] S. Cierjacks, P. Forti, D. Kopsch, L. Kropp, J. Nebe, H. Unseld, High Resolution Total Neutron Cross Sections Between 0.5 TO $30 \mathrm{MeV}$. (1968)

[11] S. Cierjacks, F. Hinterberger, G. Schmalz, D. Erbe, P. Rossen, B. Leugers, Nuclear Instruments and Methods 169, 185 (1980)

[12] R. Sayer, L. Leal, N. Larson, R. Spencer, R. Wright, Journal of Nuclear Science and Technology 39, 88 (2002)

[13] C.H. Johnson, J.L. Fowler, L.A. Galloway, N.W. Hill, ORNL-4937 (1973)

[14] R. Bahran, PhD thesis, Rensselaer Polytechnic Institute (2013)

[15] R. Bahran, D. Barry, R. Block, G. Leinweber, M. Rapp, A. Daskalakis, E. Blain, D. Williams, B. McDermott, L. Leal et al., Phys. Rev. C 92, 024601 (2015)

[16] A. Borella, G. Aerts, F. Gunsing, M. Moxon, P. Schillebeeckx, R. Wynants, Nuclear Instruments and Methods in Physics Research Section A: Accelerators, Spectrometers, Detectors and Associated Equipment 577, 626 (2007)

[17] A. Daskalakis, R. Bahran, E. Blain, B. McDermott, S. Piela, Y. Danon, D. Barry, G. Leinweber, R. Block, M. Rapp et al., Annals of Nuclear Energy 73, 455 (2014)

[18] JEFF-3.2 NEA Data Bank (2012)

[19] K. Shibata, O. Iwamoto, T. Nakagawa, N. Iwamoto, A. Ichihara, S. Kunieda, S. Chiba, K. Furutaka, N. Otuka, T. Ohasawa et al., Journal of Nuclear Science and Technology 48, 1 (2011) 